\title{
Distributive Lattices, Bipartite Graphs and Alexander Duality
}

\begin{abstract}
A certain squarefree monomial ideal $H_{P}$ arising from a finite partially ordered set $P$ will be studied from viewpoints of both commutative algbera and combinatorics. First, it is proved that the defining ideal of the Rees algebra of $H_{P}$ possesses a quadratic Gröbner basis. Thus in particular all powers of $H_{P}$ have linear resolutions. Second, the minimal free graded resolution of $H_{P}$ will be constructed explicitly and a combinatorial formula to compute the Betti numbers of $H_{P}$ will be presented. Third, by using the fact that the Alexander dual of the simplicial complex $\Delta$ whose Stanley-Reisner ideal coincides with $H_{P}$ is Cohen-Macaulay, all the Cohen-Macaulay bipartite graphs will be classified.
\end{abstract}

\section{Introduction}

Let $P$ be a finite partially ordered set (poset for short) and write $\mathcal{J}(P)$ for the finite poset which consists of all poset ideals of $P$, ordered by inclusion. Here a poset ideal of $P$ is a subset $I$ of $P$ such that if $x \in I, y \in P$ and $y \leq x$, then $y \in I$. In particular the empty set as well as $P$ itself is a poset ideal of $P$. It follows easily that $\mathcal{J}(P)$ is a finite distributive lattice [12, p. 106]. Conversely, Birkhoff's fundamental structure theorem [12, Theorem 3.4.1] guarantees that, for any finite distributive lattice $\mathcal{L}$, there exists a unique poset $P$ such that $\mathcal{L}=\mathcal{J}(P)$.

Let $P$ be a finite poset with $|P|=n$, where $|P|$ is the cardinality of $P$, and let $S=K\left[\left\{x_{p}, y_{p}\right\}_{p \in P}\right]$ denote the polynomial ring in $2 n$ variables over a field $K$ with each $\operatorname{deg} x_{p}=\operatorname{deg} y_{p}=1$.

We associate each poset ideal $I$ of $P$ with the squarefree monomial

$$
u_{I}=\left(\prod_{p \in I} x_{p}\right)\left(\prod_{p \in P \backslash I} y_{p}\right)
$$

of $S$ of degree $n$. In particular $u_{P}=\prod_{p \in P} x_{p}$ and $u_{\emptyset}=\prod_{p \in P} y_{p}$. 
The normal affine semigroup ring $K\left[\left\{u_{I}\right\}_{I \in \mathcal{J}(P)}\right]$ is studied in [9] from viewpoints of both commutative algebra and combinatorics.

In the present paper, however, we are interested in the squarefree monomial ideal

$$
H_{P}=\left(\left\{u_{I}\right\}_{I \in \mathcal{J}(P)}\right)
$$

of $S$ generated by all $u_{I}$ with $I \in \mathcal{J}(P)$.

The outline of the present paper is as follows. First, in Section 1 we study the Rees algebra $\mathcal{R}\left(H_{P}\right)$ of $H_{P}$ and establish our fundamental Theorem 1.1 which says that the defining ideal of $\mathcal{R}\left(H_{P}\right)$ possesses a reduced Gröbner basis consisting of quadratic binomials whose initial monomials are squarefree. Thus $\mathcal{R}\left(H_{P}\right)$ turns out to be normal and Koszul (Corollary 1.2), and all powers of $H_{P}$ have linear resolutions (Corollary 1.3).

Second, in Section 2 the minimal graded free $S$-resolution of $H_{P}$ is constructed explicitly. See Theorem 2.1. The resolution tells us how to compute the Betti numbers $\beta_{i}\left(H_{P}\right)$ of $H_{P}$ in terms of the combinatorics of the distributive lattice $\mathcal{L}=\mathcal{J}(P)$. In fact, if $b_{i}(\mathcal{L})$ is the number of intervals $[I, J]$ of $\mathcal{L}=\mathcal{J}(P)$ which are Boolean lattices of rank $i$, then the $i$ th Betti number $\beta_{i}\left(H_{P}\right)$ of $H_{P}$ coincides with $b_{i}(\mathcal{L})$. See Corollary 2.2. (A Boolean lattice of rank $i$ is the distributive lattice $B_{i}$ which consists of all subsets of $\{1, \ldots, i\}$, ordered by inclusion.) Thus in particular for a finite distributive lattice $\mathcal{L}=\mathcal{J}(P)$, one has $\sum_{i \geq 0}(-1)^{i} b_{i}(\mathcal{L})=1$. See Corollary 2.3. In addition, it is shown that the ideal $H_{P}$ is of height 2 and a formula to compute the multiplicity of $S / H_{P}$ will be given. See Proposition 2.4 (and Corollary 2.5).

Let $\Delta_{P}$ denote the simplicial complex on the vertex set $\left\{x_{p}, y_{p}\right\}_{p \in P}$ such that the squarefree monomial ideal $H_{P}$ coincides with the Stanley-Reisner ideal $I_{\Delta_{P}}$. In Section 3 the Alexander dual $\Delta_{P}^{\vee}$ of $\Delta_{P}$ will be studied. Since the Stanley-Reisner ideal $H_{P}=I_{\Delta_{P}}$ has a linear resolution, it follows from [4, Theorem 3] that $\Delta_{P}^{\vee}$ is Cohen-Macaulay. It will turn out that the Stanley-Reisner ideal $I_{\Delta_{P}^{\vee}}$ of $\Delta_{P}^{\vee}$ is an edge ideal of a finite bipartite graph. Somewhat surprisingly, this simple observation enables us to classify all Cohen-Macaulay bipartite graphs. In fact, Theorem 3.4 says that a finite bipartite graph $G$ is Cohen-Macaulay if and only if $G$ comes from the comparability graph of a finite poset.

\section{Monomial ideals arising from distributive lattices}

Work with the same notation as in Introduction. Let $P$ be a finite poset with $|P|=n$ and $S=K\left[\left\{x_{p}, y_{p}\right\}_{p \in P}\right]$ the polynomial ring in $2 n$ variables over a field $K$ with each $\operatorname{deg} x_{p}=$ $\operatorname{deg} y_{p}=1$. Recall that we associate each poset ideal $I$ of $P$ with the squarefree monomial $u_{I}=\left(\prod_{p \in I} x_{p}\right)\left(\prod_{p \in P \backslash I} y_{p}\right)$ of $S$ of degree $n$, and introduce the ideal $H_{P}=\left(\left\{u_{I}\right\}_{I \in \mathcal{J}(P)}\right)$ of $S$.

Let $\mathcal{R}\left(H_{P}\right)$ denote the Rees algebra of $H_{P}$ and $\mathcal{W}_{P}$ the defining ideal of $\mathcal{R}\left(H_{P}\right)$. In other words, $\mathcal{R}\left(H_{P}\right)$ is the affine semigroup ring

$$
\mathcal{R}\left(H_{P}\right)=K\left[\left\{x_{p}, y_{p}\right\}_{p \in P},\left\{u_{I} t\right\}_{I \in \mathcal{J}(P)}\right] \quad\left(\subset K\left[\left\{x_{p}, y_{p}\right\}_{p \in P}, t\right]\right)
$$


and $\mathcal{W}_{P}$ is the kernel of the surjective ring homomorphism $\varphi: K[\mathbf{x}, \mathbf{y}, \mathbf{z}] \rightarrow \mathcal{R}\left(H_{P}\right)$, where

$$
K[\mathbf{x}, \mathbf{y}, \mathbf{z}]=K\left[\left\{x_{p}, y_{p}\right\}_{p \in P},\left\{z_{I}\right\}_{I \in \mathcal{J}(P)}\right]
$$

is the polynomial ring over $K$ and where $\varphi$ is defined by setting $\varphi\left(x_{p}\right)=x_{p}, \varphi\left(y_{p}\right)=y_{p}$ and $\varphi\left(z_{I}\right)=u_{I} t$.

For the convenience of our discussion, in the remainder of the present section, we will use the notation $P=\left\{p_{1}, \ldots, p_{n}\right\}$ and write $x_{i}, y_{i}$ instead of $x_{p_{i}}, y_{p_{i}}$. Let $<_{l e x}$ denote the lexicographic order [5, p. 329] on $S$ induced by the ordering $x_{1}>\cdots>x_{n}>y_{1}>\cdots>y_{n}$ and $<^{\sharp}$ the reverse lexicographic order [5, p. 330] on $K\left[\left\{z_{I}\right\}_{I \in \mathcal{J}(P)}\right]$ induced by an ordering of the variables $z_{I}$ 's such that $z_{I}>z_{J}$ if $J \subset I$ in $\mathcal{J}(P)$. We then introduce the new monomial order $<_{\text {lex }}^{\sharp}$ on $T$ by setting

$$
\left(\prod_{i=1}^{n} x_{i}^{a_{i}} y_{i}^{b_{i}}\right)\left(z_{I_{1}} \cdots z_{I_{q}}\right)<_{l e x}^{\sharp}\left(\prod_{i=1}^{n} x_{i}^{a_{i}^{\prime}} y_{i}^{b_{i}^{\prime}}\right)\left(z_{I_{1}^{\prime}} \cdots z_{I_{q^{\prime}}^{\prime}}\right)
$$

if either

(i) $\prod_{i=1}^{n} x_{i}^{a_{i}} y_{i}^{b_{i}}<_{l e x} \prod_{i=1}^{n} x_{i}^{a_{i}^{\prime}} y_{i}^{b_{i}^{\prime}}$

or

(ii) $\quad \prod_{i=1}^{n} x_{i}^{a_{i}} y_{i}^{b_{i}}=\prod_{i=1}^{n} x_{i}^{a_{i}^{\prime}} y_{i}^{b_{i}^{\prime}}$ and $z_{I_{1}} \cdots z_{I_{q}}<^{\sharp} z_{I_{1}^{\prime}} \cdots z_{I_{q^{\prime}}^{\prime}}$.

Theorem 1.1 The reduced Gröbner basis $\mathcal{G}_{<^{\sharp}}\left(\mathcal{W}_{P}\right)$ of the defining ideal $\mathcal{W}_{P} \subset K[\mathbf{x}, \mathbf{y}, \mathbf{z}]$ with respect to the monomial order $<_{\operatorname{lex}}^{\sharp}$ consists of quadratic binomials whose initial monomials are squarefree.

Proof: The reduced Gröbner basis of $\mathcal{W}_{P} \cap K\left[\left\{z_{I}\right\}_{I \in \mathcal{J}(P)}\right]$ with respect to the reverse lexicographic order $<^{\sharp}$ coincides with $\mathcal{G}_{<_{\text {lex }}^{\sharp}}\left(\mathcal{W}_{P}\right) \cap K\left[\left\{z_{I}\right\}_{I \in \mathcal{J}(P)}\right]$. It follows from [9] that $\mathcal{G}_{<_{\text {lex }}^{\sharp}}\left(\mathcal{W}_{P}\right) \cap K\left[\left\{z_{I}\right\}_{I \in \mathcal{J}(P)}\right]$ consists of those binomials

$$
z_{I} z_{J}-z_{I \wedge J} z_{I \vee J}
$$

such that $I$ and $J$ are incomparable in the distributive lattice $\mathcal{J}(P)$.

It is known [14, Corollary 4.4] that the reduced Gröbner basis of $\mathcal{W}_{P}$ consists of irreducible binomials of $K[\mathbf{x}, \mathbf{y}, \mathbf{z}]$. Let

$$
f=\left(\prod_{i=1}^{n} x_{i}^{a_{i}} y_{i}^{b_{i}}\right)\left(z_{I_{1}} \cdots z_{I_{q}}\right)-\left(\prod_{i=1}^{n} x_{i}^{a_{i}^{\prime}} y_{i}^{b_{i}^{\prime}}\right)\left(z_{I_{1}^{\prime}} \cdots z_{I_{q}^{\prime}}\right)
$$


be an irreducible binomial of $K[\mathbf{x}, \mathbf{y}, \mathbf{z}]$ belonging to $\mathcal{G}_{<_{\text {lex }}^{\sharp}}\left(\mathcal{W}_{P}\right)$ with

$$
\left(\prod_{i=1}^{n} x_{i}^{a_{i}} y_{i}^{b_{i}}\right)\left(z_{I_{1}} \cdots z_{I_{q}}\right)
$$

its initial monomial, where $z_{I_{1}} \leq \cdots \leq z_{I_{q}}$ and $z_{I_{1}^{\prime}} \leq \cdots \leq z_{I_{q}^{\prime}}$.

Let $f \notin K\left[\left\{z_{I}\right\}_{I \in \mathcal{J}(P)}\right]$. Let $j$ denote an integer for which $I_{j}^{\prime} \not \subset I_{j}$. Such an integer exists. In fact, if $I_{j}^{\prime} \subset I_{j}$ for all $j$, then each $a_{i}=0$ and each $b_{i}^{\prime}=0$. This is impossible since $\left(\prod_{i=1}^{n} x_{i}^{a_{i}} y_{i}^{b_{i}}\right)\left(z_{I_{1}} \cdots z_{I_{q}}\right)$ is the initial monomial of $f$.

Let $p_{i} \in I_{j}^{\prime} \backslash I_{j}$. Then $p_{i}$ belongs to each of $I_{j}^{\prime}, I_{j+1}^{\prime}, \ldots, I_{q}^{\prime}$, and does not belong to each of $I_{1}, I_{2}, \ldots, I_{j}$. Hence $a_{i}>0$.

Let $p_{i_{0}} \in P$ with $p_{i_{0}} \in I_{j}^{\prime} \backslash I_{j}$ such that $I_{j} \cup\left\{p_{i_{0}}\right\} \in \mathcal{J}(P)$. Thus $a_{i_{0}}>0$. Let $J=I_{j} \cup\left\{p_{i_{0}}\right\}$. Then the binomial $g=x_{i_{0}} z_{I_{j}}-y_{i_{0}} z_{J}$ belongs to $\mathcal{W}_{P}$ with $x_{i_{0}} z_{I_{j}}$ its initial monomial. Since $x_{i_{0}} z_{I_{j}}$ divides the initial monomial of $f$, it follows that the initial monomial of $f$ must coincides with $x_{i_{0}} z_{I}$, as desired.

It is well known that a homogeneous affine semigroup ring whose defining ideal has an initial ideal which is generated by squarefree (resp. quadratic) monomials is normal (resp. Koszul). See, e.g., [14, Proposition 13.15] and [6].

Corollary 1.2 Let $P$ be an arbitrary finite poset. Then the Rees algebra $\mathcal{R}\left(H_{P}\right)$ is normal and Koszul.

On the other hand, Stefan Blum [2] proved that if the Rees algebra of an ideal is Koszul, then all powers of the ideal have linear resolutions.

Corollary 1.3 Let $P$ be an arbitrary finite poset. Then all powers of $H_{P}$ have linear resolutions.

\section{The free resolution and Betti numbers of $H_{P}$}

Corollary 1.3 says that the monomial ideal $H_{P}$ arising from a finite poset $P$ has a linear resolution. The main purpose of the present section is to construct a minimal graded free $S$-resolution $\mathbb{F}=\mathbb{F}_{P}$ of $H_{P}$ explicitly.

Let $P$ be a finite poset with $|P|=n$ and $S=K\left[\left\{x_{p}, y_{p}\right\}_{p \in P}\right]$ the polynomial ring in $2 n$ variables over a field $K$ with each $\operatorname{deg} x_{p}=\operatorname{deg} y_{p}=1$. Recall that, for each poset ideal $I$ of $P$, we associate the squarefree monomial $u_{I}=\left(\prod_{p \in I} x_{p}\right)\left(\prod_{p \in P \backslash I} y_{p}\right)$ of $S$ of degree $n$. Let $H_{P}$ denote the ideal of $S$ generated by all $u_{I}$ with $I \in \mathcal{J}(P)$.

The maximal elements of a poset ideal $I$ of $P$ are called the generators of $I$. Let $M(I)$ denote the set of generators of $I$.

The construction of a minimal graded free $S$-resolution $\mathbb{F}=\mathbb{F}_{P}$ of $H_{P}$ is achieved as follows: For all $i \geq 0$ let $\mathbb{F}_{i}$ denote the free $S$-module with basis

$$
e(I, T)
$$


where

$$
I \in \mathcal{J}(P), T \subset P, I \cap T \subset M(I),|I \cap T|=i \quad \text { and } \quad|I \cup T|=n+i .
$$

Extending the partial order on $P$ to a total order, we define for $i>0$ the differential

$$
\partial: \mathbb{F}_{i} \rightarrow \mathbb{F}_{i-1}
$$

by

$$
\partial(e(I, T))=\sum_{p \in I \cap T}(-1)^{\sigma(I \cap T, p)}\left(x_{p} e(I \backslash\{p\}, T)-y_{p} e(I, T \backslash\{p\})\right),
$$

where for a subset $Q \subset P$ and $p \in Q$ we set $\sigma(Q, p)=|\{q \in Q: q<p\}|$.

With the notation introduced we have

Theorem 2.1 The complex $\mathbb{F}$ is a graded minimal free $S$-resolution of $H_{P}$.

Proof: We define an augmentation $\varepsilon: \mathbb{F}_{0} \rightarrow H_{P}$ by setting

$$
\varepsilon(e(I, T))=u_{I}
$$

for all $e(I, T) \in \mathbb{F}_{0}$. Note that if $e(I, T)$ is a basis element of $\mathbb{F}_{0}$, then $T=[n] \backslash I$, so that $\varepsilon$ is well defined.

We first show that

$$
\cdots \stackrel{\partial}{\longrightarrow} \mathbb{F}_{1} \stackrel{\partial}{\longrightarrow} \mathbb{F}_{0} \stackrel{\varepsilon}{\longrightarrow} H_{P} \longrightarrow 0
$$

is a complex.

Let $e(I, T) \in \mathbb{F}_{1}$ with $I \cap T=\{p\}$. Then

$$
\begin{aligned}
(\varepsilon \circ \partial)(e(I, T)) & =x_{p} \varepsilon(e(I \backslash\{p\}, T))-y_{p} \varepsilon(e(I, T \backslash\{p\})) \\
& =x_{p} u_{I \backslash\{p\}}-y_{p} u_{I}=0 .
\end{aligned}
$$

Thus $\partial \circ \varepsilon=0$, as desired. 
Next we show that $\partial \circ \partial=0$. Let $e(I, T) \in \mathbb{F}_{i+1}$ and set $L=I \cap T$. Then

$$
\begin{aligned}
\partial \circ & \partial(e(I, T)) \\
= & \sum_{p \in L}(-1)^{\sigma(L, p)}\left(x_{p} \partial(e(I \backslash\{p\}, T))-y_{p} \partial(e(I, T \backslash\{p\}))\right. \\
= & \sum_{p \in L}(-1)^{\sigma(L, p)}\left[x _ { p } \left(\sum_{q \in L, q \neq p}(-1)^{\sigma(L \backslash\{p\}, q)}\right.\right. \\
& \left.\times\left(x_{q} e(I \backslash\{p, q\}, T)-y_{q} e(I \backslash\{p\}, T \backslash\{q\})\right)\right) \\
& \left.-y_{p}\left(\sum_{q \in L, q \neq p}(-1)^{\sigma(L \backslash\{p\}, q)}\left(x_{q} e(I \backslash\{q\}, T \backslash\{p\})-y_{q} e(I, T \backslash\{p, q\})\right)\right)\right] \\
= & \sum_{p, q \in L, p \neq q}(-1)^{\sigma(L, p)+\sigma(L \backslash\{p\}, q)} x_{p} x_{q} e(I \backslash\{p, q\}, T) \\
& -\sum_{p, q \in L, p \neq q}(-1)^{\sigma(L, p)+\sigma(L \backslash\{p\}, q)} x_{p} y_{q} e(I \backslash\{p\}, T \backslash\{q\}) \\
& -\sum_{p, q \in L, p \neq q}(-1)^{\sigma(L, p) \sigma(L \backslash\{p\}, q)} x_{q} y_{p} e(I \backslash\{q\}, T \backslash\{p\}) \\
& +\sum_{p \in L, p \neq q}(-1)^{\sigma(L, p)+\sigma(L \backslash\{p\}, q)} y_{p} y_{q} e(I, T \backslash\{p, q\}) \\
= & 0 .
\end{aligned}
$$

The last equality holds since $(-1)^{\sigma(L, p)+\sigma(L \backslash\{p\}, q)}=-(-1)^{\sigma(L, q)+\sigma(L \backslash\{q\}, p)}$. In order to prove that the augmented complex

$$
\cdots \longrightarrow \mathbb{F}_{1} \stackrel{\partial}{\longrightarrow} \mathbb{F}_{0} \stackrel{\varepsilon}{\longrightarrow} H_{P} \longrightarrow 0
$$

is exact we show:

(1) $H_{0}(\mathbb{F})=H_{P}$,

(2) $\mathbb{F}$ is acyclic.

For the proof of (1) we note that the Taylor relations

$$
r_{I, J}=x_{J \backslash I} y_{I \backslash J} e(I)-x_{I \backslash J} y_{J \backslash I} e(J), \quad I, J \in \mathcal{J}(P)
$$

generate the first syzygy module of $H_{P}$. Here we set for simplicity $e(I)$ for the basis element $e(I, P \backslash I)$ in $\mathbb{F}_{0}$, and denote by $x_{A} y_{B}$ the monomial $\prod_{p \in A} x_{p} \prod_{q \in B} y_{q}$.

Observe that

$$
r_{I, J}=x_{J \backslash I} r_{I, I \cap J}-x_{I \backslash J} r_{J, I \cap J} .
$$


Hence it suffices to show that $r_{I, J} \in \partial\left(\mathbb{F}_{1}\right)$ for all $I, J \in L$ with $J \subset I$. To this end we choose a sequence $J=I_{0} \subset I_{1} \subset \ldots I_{m-1} \subset I_{m}=I$ of poset ideals such that $I_{j}=I_{j-1} \cup\left\{p_{j}\right\}$ for $j=1, \ldots, m$. Then

$$
r_{I, J}=\sum_{j=1}^{m}\left(\prod_{k=j+1}^{m} x_{p_{k}} \prod_{k=1}^{j-1} y_{p_{k}}\right) r_{I_{j}, I_{j-1}}
$$

The assertion follows since $r_{I_{j}, I_{j-1}}=-\partial\left(e\left(I_{j}, P \backslash I_{j-1}\right)\right)$ for all $j$.

We prove (2), that is, the acyclicity of $\mathbb{F}$ by induction on $|P|$. If $P=\{p\}$, then $H_{P}=$ $\left(x_{p}, y_{p}\right)$, and $\mathbb{F}$ can be identified with the Koszul complex associated with $\left\{x_{p}, y_{p}\right\}$, and hence is acyclic.

Suppose now that $|P|>1$. Let $q \in P$ be a maximal element and let $Q$ be the subposet $P \backslash\{q\}$. We define a map

$$
\phi: \mathbb{F}_{Q} \rightarrow \mathbb{F}_{P}, \quad e_{i}(I, T) \mapsto e_{i}(I, T \cup\{q\})
$$

It is clear that $\phi$ is an injective map of complexes whose induced map $H_{Q}=H_{0}\left(\mathbb{F}_{Q}\right) \rightarrow$ $H_{0}\left(\mathbb{F}_{P}\right)=H_{P}$ is multiplication by $y_{q}$. Let $\mathbb{G}$ be the quotient complex $\mathbb{F}_{P} / \mathbb{F}_{Q}$. Since the multiplication map is injective, the short exact sequence of complexes

$$
0 \longrightarrow \mathbb{F}_{Q} \longrightarrow \mathbb{F}_{P} \longrightarrow \mathbb{G} \longrightarrow 0
$$

induces the long exact homology sequence

$$
\cdots \longrightarrow H_{2}(\mathbb{G}) \longrightarrow H_{1}\left(\mathbb{F}_{Q}\right) \longrightarrow H_{1}\left(\mathbb{F}_{P}\right) \longrightarrow H_{1}(\mathbb{G}) \longrightarrow 0
$$

By induction hypothesis, $H_{i}\left(\mathbb{F}_{Q}\right)=0$ for $i>0$. Hence it suffices to show that $H_{i}(\mathbb{G})=0$ for $i>0$.

The principal order ideal $(q)$ consists of all $p \in P$ with $p \leq p$. Let $R$ be the subposet $P \backslash(q)$, and let $\mathbb{C}$ be the mapping cone of the complex homomorphism

$$
\mathbb{F}_{R} \stackrel{-y_{q}}{\longrightarrow} \mathbb{F}_{R}
$$

Then we get an exact sequence

$$
0 \longrightarrow \mathbb{F}_{R} \longrightarrow \mathbb{C} \longrightarrow \mathbb{F}_{R}[-1] \longrightarrow 0
$$

Here $\mathbb{F}_{R}[-1]$ is the complex $\mathbb{F}_{R}$ shifted to the 'left', that is, $\left(\mathbb{F}_{R}[-1]\right)_{i}=\left(\mathbb{F}_{R}\right)_{i-1}$ for all $i$. By our induction hypothesis $\mathbb{F}_{R}$ is acyclic. Thus from the long exact sequence

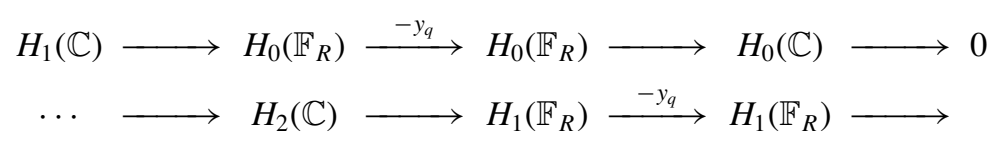


we deduce that $H_{i}(\mathbb{C})=0$ for $i>1$. We also get $H_{1}(\mathbb{C})=0$, since $H_{0}\left(\mathbb{F}_{R}\right)=H_{R}$, and since multiplication by $y_{q}$ is injective on $H_{R}$. Thus we see that $\mathbb{C}$ is acyclic.

We now claim that $\mathbb{C} \cong \mathbb{G}$, thereby proving that $\mathbb{G}$ is acyclic, as desired.

In order to prove this claim we first notice that $\mathbb{C}_{i}=\left(\mathbb{F}_{R}\right)_{i-1} \oplus\left(\mathbb{F}_{R}\right)_{i}$ for $i \geq 0$ (where $\left(\mathbb{F}_{R}\right)_{-1}=0$ ). Thus if $r=|R|$, then $\mathbb{C}_{i}$ has the basis $\mathcal{C}_{i}=\mathcal{B}_{i-1} \cup \mathcal{B}_{i}$, where

$$
\mathcal{B}_{i}=\{e(I, T): I \in L(R), T \subset R, I \cap T \subset M(I),|I \cap T|=i,|I \cup T|=r+i\} .
$$

On the other hand $\mathbb{G}_{i}$ has the basis

$$
\begin{aligned}
\mathcal{G}_{i}= & \{e(I, T): I \in L(P),(q) \subset I, T \subset P, I \cap T \subset M(I),|I \cap T|=i, \\
& |I \cup T|=n+i\} .
\end{aligned}
$$

Let $\psi_{i}: \mathbb{C}_{i} \rightarrow \mathbb{G}_{i}$ be the $S$-linear homomorphism with

$$
\psi_{i}(e(I, T))= \begin{cases}e(I \cup(q), T \cup\{q\}) & \text { if } e(I, T) \in \mathcal{B}_{i-1} \\ e(I \cup(q), T) & \text { if } e(I, T) \in \mathcal{B}_{i} .\end{cases}
$$

It is easy to see that all $\psi_{i}$ are bijections and induce an isomorphism of complexes.

Suppose $P$ is of cardinality $n$ and $P$ is an antichain, i.e., any two elements of $P$ are incomparable. Then $B_{n}=\mathcal{J}(P)$ is called the Boolean lattice of rank $n$.

Let now $\mathcal{L}$ be an arbitrary finite distributive lattice, and let $I, J \in \mathcal{L}$ with $I \leq J$. Then the set

$$
[I, J]=\{M \in \mathcal{L}: I \leq M \leq J\}
$$

is called an interval in $\mathcal{L}$. The interval $[I, J]$ with the induced partial order is again a distributive lattice. Let $b_{i}(\mathcal{L})$ denote the number of intervals of $\mathcal{L}$ which are isomorphic to Boolean lattices of rank $i$. In particular, $b_{0}(\mathcal{L})=|\mathcal{L}|$. These numbers have an algebraic interpretation.

Recall that for a graded $S$-module $M$,

$$
\beta_{i}(M)=\operatorname{dim}_{K} \operatorname{Tor}_{i}^{S}(M, K)
$$

is called the $i$ th Betti-number of $M$. If $\mathbb{F}$ is a graded minimal free resolution of $M$, then $\beta_{i}(M)$ is nothing but the rank of $\mathbb{F}_{i}$.

Corollary 2.2 Let $P$ be a finite poset, $\mathcal{L}=\mathcal{J}(P)$ the distributive lattice and $H_{P}$ the squarefree monomial ideal arising from $P$. Then

(a) $b_{i}(\mathcal{L})=\beta_{i}\left(H_{P}\right)$ for all $i$;

(b) the following three numbers are equal: 
(i) the projective dimension of $H_{P}$;

(ii) the maximum of the ranks of Boolean lattices which are isomorphic to an interval of $\mathcal{L}$;

(iii) the Sperner number of $P$, i.e., the maximum of the cardinalities of antichains of $P$.

Proof: (a) For each $i \geq 0$, let $\mathcal{J}_{i}$ be the set of pairs $(I, S)$, where $I \in \mathcal{L}, S \subset M(I)$ and $|S|=i$, and let $\mathcal{B}_{i}$ be the set of basis elements $e(I, T)$ of $\mathbb{F}_{i}$. Then

$$
\mathcal{B}_{i} \longrightarrow \mathcal{J}_{i}, \quad e(I, T) \mapsto(I, I \cap T)
$$

establishes a bijection between these two sets.

Since for each $(I, S) \in \mathcal{J}_{i}$, the elements in $S$ are pairwise incomparable it is clear that $[I \backslash S, I]$ is isomorphic to a Boolean lattice of rank $i$.

Conversely, suppose $[J, I]$ is isomorphic to a Boolean lattice of rank $i$. Then $S=I \backslash J$ is of a set of cardinality $i$, and $J \cup T \in \mathcal{L}$ for all subsets $T \subset S$.

Suppose that $S \not \subset M(I)$. Then there exists, $q \in S$ and $p \in I$ such that $p>q$. If $p \in J$, then $q \in J$, a contradiction. Thus $p \in S$, and hence $(J, p) \in \mathcal{L}$. This is again a contradiction, because it would imply that $q \in(J, p)$. Hence we have shown that $(I, S) \in \mathcal{J}_{i}$.

It follows that the assignment $e(I, T) \mapsto[I \backslash(I \cap T), I]$ establishes a bijection between the basis of $\mathbb{F}_{i}$ and the intervals of $[J, I]$ in $\mathcal{L}$ which are isomorphic to Boolean lattices.

(b) is an immediate consequence of (a) and its proof.

Corollary 2.3 Let $\mathcal{L}$ be a finite distributive lattice. Then

$$
\sum_{i \geq 0}(-1)^{i+1} b_{i}(\mathcal{L})=1
$$

Corollary 2.3 is a special case of [12, Exercise 3.19 (b)] and the resolution constructed in Theorem 2.1 is the cellular resolution [1] of the cubical complex appearing in Topological Remark [12, pp. 178-179]. In the forthcoming paper [8], we construct such the resolutions in more general contexts and show that these resolutions are cellular in some cases.

Let $\Delta_{P}$ be the simplicial complex attached to the squarefree monomial ideal $H_{P}$. In the next section we will see (Lemma 3.1) that the Stanley-Reisner ideal attached to the Alexander dual $\Delta_{P}^{\vee}$ is generated by the monomials $x_{p} y_{q}$ such that $p \leq q$. Hence for the Stanley-Reisner ideal of $\Delta_{P}$ we have

$$
I_{\Delta_{P}}=\bigcap_{p, q \in P, p \leq q}\left(x_{p}, y_{q}\right)
$$

In particular we get

Proposition 2.4 Let $P$ be a finite poset. Then the squarefree monomial ideal $H_{P}$ is of height 2 , and the multiplicity of $S / H_{P}$ is given by

$$
e\left(S / H_{P}\right)=|\{(p, q): p, q \in P, p \leq q\}| .
$$


Let $I \subset S$ be an arbitrary graded ideal with graded minimal free resolution

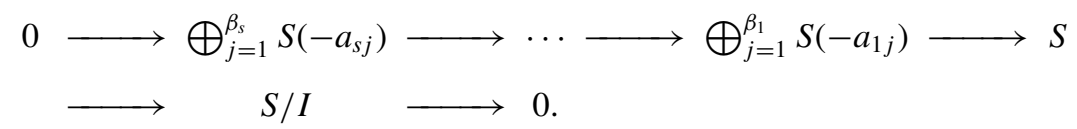

Suppose the height of $I$ equals $h$. Then by a formula of Peskine and Szpiro [11] one has

$$
e(S / I)=\frac{(-1)^{h}}{h !} \sum_{i=1}^{s}(-1)^{i} \sum_{j=1}^{\beta_{i}} a_{i j}^{h}
$$

Applying this formula in our situation and using Corollary 2.2 and Proposition 2.4 we get

Corollary 2.5 Let $P$ be a finite poset with $|P|=n$, and let $\mathcal{L}=\mathcal{J}(P)$ be the distributive lattice. Then

$$
|\{(p, q): p, q \in P, p \leq q\}|=\frac{1}{2} \sum_{i \geq 0}(-1)^{i+1} b_{i}(\mathcal{L})(n+i)^{2} .
$$

We close this section with an example. Let $P$ be the poset with Hasse diagram

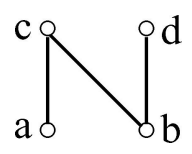

The distributive lattice $\mathcal{L}=\mathcal{J}(P)$ has the Hasse diagram

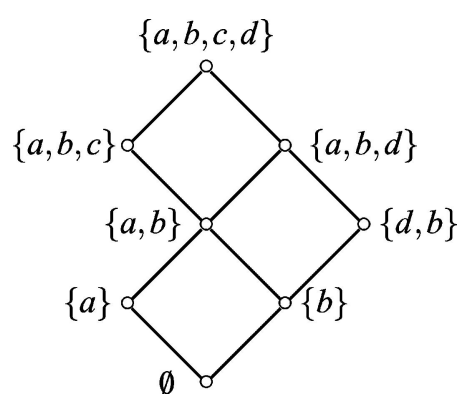

Thus $H_{P}=(u v w x, a v w x, b u w x, a b w x, b d u w, a b c x, a b d w, a b c d)$. Here we use for convenience the indeterminates $a, b, c, d, u, v, w, x$ instead of $x_{p}$ and $y_{p}$. The free resolution 
of $H_{P}$ is given by

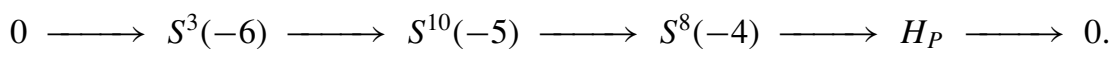

We see from the Hasse diagram that the $i$ th Betti number of $H_{P}$ coincides with number of intervals of $\mathcal{L}$ which are isomorphic to Boolean lattices of rank $i$. The number of pairs $(p, q)$ in the poset $P$ with $p \leq q$ is equal to 7 , and this is also the number we get from Corollary 2.5 , namely $(1 / 2)(-8 \cdot 16+10 \cdot 25-3 \cdot 36)=7$.

\section{Alexander duality and Cohen-Macaulay bipartite graphs}

We refer the reader to, e.g., $[3,10,13]$ for fundamental information about Stanley-Reisner rings.

Let $P=\left\{p_{1}, \ldots, p_{n}\right\}$ be a finite poset and $S=K\left[x_{1}, \ldots, x_{n}, y_{1}, \ldots, y_{n}\right]$ the polynomial ring in $2 n$ variables over a field $K$ with each $\operatorname{deg} x_{i}=\operatorname{deg} y_{i}=1$. We will use the notation $x_{i}, y_{i}$ instead of $x_{p_{i}}, y_{p_{i}}$, and set $V_{n}=\left\{x_{1}, \ldots, x_{n}, y_{1}, \ldots, y_{n}\right\}$.

Recall that $H_{P}$ is the ideal of $S$ which is generated by those squarefree monomials $u_{I}=\left(\prod_{p_{i} \in I} x_{i}\right)\left(\prod_{p_{i} \in P \backslash I} y_{i}\right)$ with $I \in \mathcal{J}(P)$. It then follows that there is a unique simplicial complex $\Delta_{P}$ on $V_{n}$ such that the Stanley-Reisner ideal $I_{\Delta_{P}}$ coincides with $H_{P}$. We study the Alexander dual $\Delta_{P}^{\vee}$ of $\Delta_{P}$, which is the simplicial complex

$$
\Delta_{P}^{\vee}=\left\{V_{n} \backslash F: F \notin \Delta_{P}\right\}
$$

on $V_{n}$.

Lemma 3.1 The Stanley-Reisner ideal of $\Delta_{P}^{\vee}$ is generated by those squarefree quadratic monomials $x_{i} y_{j}$ such that $p_{i} \leq p_{j}$ in $P$.

Proof: Let $w=x_{1} \ldots x_{n} y_{1} \ldots y_{n}$. If $u$ is a squarefree monomial of $S$, then we write $\operatorname{supp}(u)$ for the support of $u$, i.e., $\operatorname{supp}(u)=\left\{x_{i}: x_{i}\right.$ divides $\left.u\right\} \cup\left\{y_{j}: y_{j}\right.$ divides $\left.u\right\}$. Now since $\left\{\operatorname{supp}\left(u_{I}\right): I \in \mathcal{J}(P)\right\}$ is the set of minimal nonfaces of $\Delta_{P}$, it follows that $\left\{\operatorname{supp}\left(w / u_{I}\right): I \in \mathcal{J}(P)\right\}$ is the set of facets (maximal faces) of $\Delta_{P}^{\vee}$. Our work is to find the minimal nonfaces of $\Delta_{P}^{\vee}$. Since $\operatorname{supp}\left(w / u_{\emptyset}\right)=x_{1} \cdots x_{n}$ and $\operatorname{supp}\left(w / u_{P}\right)=y_{1} \cdots y_{n}$, both $\left\{x_{1}, \ldots, x_{n}\right\}$ and $\left\{y_{1}, \ldots, y_{n}\right\}$ are faces of $\Delta_{P}^{\vee}$. Let $F \subset V_{n}$ be a nonfaces of $\Delta_{P}^{\vee}$. Let $F_{x}=F \cap\left\{x_{1}, \ldots, x_{n}\right\}$ and $F_{y}=\left\{x_{j}: y_{j} \in F\right\}$. Then $F_{x} \neq \emptyset$ and $F_{y} \neq \emptyset$. Since $\left\{x_{i}, y_{i}\right\}$ is a minimal nonface of $\Delta_{P}^{\vee}$, we will assume that $F_{x} \cap F_{y}=\emptyset$. Since $F$ is a nonface, there exists no poset ideal $I$ of $P$ with $F_{x} \cap\left\{x_{i}: p_{i} \in I\right\}=\emptyset$ and $F_{y} \subset\left\{x_{i}: p_{i} \in I\right\}$. Hence there are $x_{i} \in F_{x}$ and $x_{j} \in F_{y}$ such that $p_{i}<p_{j}$. Thus $\left\{x_{i}, y_{j}\right\}$ is a nonface of $\Delta_{P}^{\vee}$. Hence the set of minimal nonfaces of $\Delta_{P}^{\vee}$ consists of those 2-element subsets $\left\{x_{i}, y_{j}\right\}$ of $V_{n}$ such that $p_{i} \leq p_{j}$ in $P$, as required.

Let $G$ be a finite graph on the vertex set $[N]=\{1, \ldots, N\}$ with no loops and no multiple edges. We will assume that $G$ possesses no isolated vertex, i.e., for each vertex $i$ there is an edge $e$ of $G$ with $i \in e$. A vertex cover of $G$ is a subset $C \subset[N]$ such that, for each edge 
$\{i, j\}$ of $G$, one has either $i \in C$ or $j \in C$. Such a vertex cover $C$ is called minimal if no subset $C^{\prime} \subset C$ with $C^{\prime} \neq C$ is a vertex cover of $G$. We say that a finite graph $G$ is unmixed if all minimal vertex covers of $G$ have the same cardinality.

Let $K[\mathbf{z}]=K\left[z_{1}, \ldots, z_{N}\right]$ denote the polynomial ring in $N$ variables over a field $K$. The edge ideal of $G$ is the ideal $I(G)$ of $K[\mathbf{z}]$ generated by those squarefree quadratic monomials $z_{i} z_{j}$ such that $\{i, j\}$ is an edge of $G$. A finite graph $G$ on $[N]$ is called Cohen-Macaulay over $K$ if the quotient ring $K[\mathbf{z}] / I(G)$ is Cohen-Macaulay. Every Cohen-Macaulay graph is unmixed ( [15, Proposition 6.1.21]).

A finite graph $G$ on $[N]$ is bipartite if there is a partition $[N]=W \cup W^{\prime}$ such that each edge of $G$ is of the form $\{i, j\}$ with $i \in W$ and $j \in W^{\prime}$. A basic fact on the graph theory says that a finite graph $G$ is bipartite if and only if $G$ possesses no cycle of odd length. A tree is a connected graph with no cycle. A tree is Cohen-Macaulay if and only if it is unmixed ( [15, Corollary 6.3.5]).

Given a finite poset $P=\left\{p_{1}, \ldots, p_{n}\right\}$, we write $G(P)$ for the bipartite graph on the vertex set $\left\{x_{1}, \ldots, x_{n}\right\} \cup\left\{y_{1}, \ldots, y_{n}\right\}$ whose edges are those $\left\{x_{i}, y_{j}\right\}$ such that $p_{i} \leq p_{j}$ in $P$. Lemma 3.1 says that the Stanley-Reisner ideal of $\Delta_{P}^{\vee}$ is equal to the edge ideal of $G(P)$. Since the Stanley-Reisner ideal $H_{P}=I_{\Delta_{P}}$ has a linear resolution, it follows from [4, Theorem 3] that $\Delta_{P}^{\vee}$ is Cohen-Macaulay. Then [15, Theorem 6.4.7] says that $\Delta_{P}^{\vee}$ is shellable. Hence $I_{\Delta_{P}}$ has linear quotients (e.g., [7]).

Corollary 3.2 The Alexander dual $\Delta_{P}^{\vee}$ is shellable and the ideal $H_{P}$ has linear quotients.

We now turn to the problem of classifying the Cohen-Macaulay bipartite graphs by using the Alexander dual $\Delta_{P}^{\vee}$.

Let $G$ be a finite bipartite graph on the vertex set $W \cup W^{\prime}$ with $W=\left\{i_{1}, \ldots, i_{s}\right\}$ and $W^{\prime}=\left\{j_{1}, \ldots, j_{t}\right\}$, where $s \leq t$. For each subset $U$ of $W$, we write $N(U)$ for the set of those vertices $j \in W^{\prime}$ for which there is a vertex $i \in U$ such that $\{i, j\}$ is an edge of $G$. The well-known "marriage theorem" in graph theory says that if $|U| \leq|N(U)|$ for all subsets $U$ of $W$, then there is a subset $W^{\prime \prime}=\left\{j_{\ell_{1}}, \ldots, j_{\ell_{s}}\right\} \subset W^{\prime}$ with $\left|W^{\prime \prime}\right|=s$ such that $\left\{i_{k}, j_{\ell_{k}}\right\}$ is an edge of $G$ for $k=1,2, \ldots, s$.

Let $G$ be a finite bipartite graph on the vertex set $W \cup W^{\prime}$ and suppose that $G$ is unmixed. Since each of $W$ and $W^{\prime}$ is a minimal vertex cover, one has $|W|=\left|W^{\prime}\right|$. Let $W=$ $\left\{x_{1}, \ldots, x_{n}\right\}$ and $W^{\prime}=\left\{y_{1}, \ldots, y_{n}\right\}$. Since $(W \backslash U) \cup N(U)$ is a vertex cover of $G$ for all subsets $U$ of $W$ and since $G$ is unmixed, it follows that $|U| \leq|N(U)|$ for all subsets $U$ of $W$. Thus the marriage theorem enables us to assume that $G$ satisfies the condition as follows: $(\sharp)\left\{x_{i}, y_{i}\right\}$ is an edge of $G$ for all $1 \leq i \leq n$.

Lemma 3.3 Work with the same notation as above and, furthermore, suppose that $G$ is a Cohen-Macaulay graph. Then, after a suitable change of the labeling of variables $y_{1}, \ldots, y_{n}$, the edge set of $G$ satisfies the condition $(\sharp)$ together with the condition as follows: $(\sharp \sharp)$ if $\left\{x_{i}, y_{j}\right\}$ is an edge of $G$, then $i \leq j$.

Proof: Let $\Delta$ be the Cohen-Macaulay complex on the vertex set $W \cup W^{\prime}$ whose StanleyReisner ideal $I_{\Delta}$ coincides with $I(G)$. Recall that every Cohen-Macaulay complex is 
strongly connected and that all links of a Cohen-Macaulay complex are again CohenMacaulay. Since both $W$ and $W^{\prime}$ are facets of $\Delta$, it follows (say, by induction on $n$ ) that, after a suitable change of the labeling of variables $x_{1}, \ldots, x_{n}$ and $y_{1}, \ldots, y_{n}$, the subset $F_{i}=\left\{y_{1}, \ldots, y_{i}, x_{i+1}, \ldots, x_{n}\right\}$ is a facet of $\Delta$ for each $0 \leq i \leq n$, where $F_{0}=W$ and $F_{n}=W^{\prime}$. In particular $\left\{x_{i}, y_{j}\right\}$ cannot be an edge of $G$ if $j<i$. In other words, the edge set of $G$ satisfies the conditions $(\sharp)$ and $(\sharp \sharp)$, as required.

Theorem 3.4 Let $G$ be a finite bipartite graph on the vertex set $W \cup W^{\prime}$, where $W=$ $\left\{x_{1}, \ldots, x_{n}\right\}$ and $W^{\prime}=\left\{y_{1}, \ldots, y_{n}\right\}$, and suppose that the edge set of $G$ satisfies the conditions $(\sharp)$ and $(\sharp \sharp)$. Then $G$ is a Cohen-Macaulay graph if and only if the following condition $(\sharp \sharp)$ is satisfied:

$(\sharp \sharp \sharp)$ If $\left\{x_{i}, y_{j}\right\}$ and $\left\{x_{j}, y_{k}\right\}$ are edges of $G$ with $i<j<k$, then $\left\{x_{i}, y_{k}\right\}$ is an edge of $G$.

Proof: ("Only if") Let $G$ be a Cohen-Macaulay graph satisfying ( $\sharp)$ and ( $\sharp)$ and $\Delta$ the Cohen-Macaulay complex on the vertex set $W \cup W^{\prime}$ whose Stanley-Reisner ideal coincides with $I(G)$. Let $\left\{x_{i}, y_{j}\right\}$ and $\left\{x_{j}, y_{k}\right\}$ be edges of $G$ with $i<j<k$ and suppose that $\left\{x_{i}, y_{k}\right\}$ is not an edge of $G$. Since every Cohen-Macaulay complex is pure and since $\left\{x_{i}, y_{k}\right\}$ is a face of $\Delta$, it follows that there is an $n$-element subset $F \subset W \cup W^{\prime}$ of $G$ with $\left\{x_{i}, y_{k}\right\} \subset F$ such that $F$ is independent in $G$, i.e., no 2-element subset of $F$ is an edge of $G$. One has $y_{j} \notin F$ and $x_{j} \notin F$ since $\left\{x_{i}, y_{j}\right\}$ and $\left\{x_{j}, y_{k}\right\}$ are edges of $G$. Since $\left\{x_{\ell}, y_{\ell}\right\}$ is an edge of $G$ for each $1 \leq \ell \leq n$, the independent subset $F$ can contain both $x_{i}$ and $y_{i}$ for no $1 \leq i \leq n$. Thus to find such an $n$-element independent set $F$ is impossible.

("If") Now, suppose that a finite bipartite graph $G$ on the vertex set $W \cup W^{\prime}$ satisfies the conditions $(\sharp),(\sharp \sharp)$ together with $(\sharp \sharp)$. Let $\leq$ denote the binary relation on $P=$ $\left\{p_{1}, \ldots, p_{n}\right\}$ defined by setting $p_{i} \leq p_{j}$ if $\left\{x_{i}, y_{j}\right\}$ is an edge of $G$. By ( $\sharp$ ) one has $p_{i} \leq p_{i}$ for each $1 \leq i \leq n$. By ( $\sharp \sharp)$ if $p_{i} \leq p_{j}$ and $p_{j} \leq p_{i}$, then $p_{i}=p_{j}$. By ( $\left.\sharp \sharp\right)$ if $p_{i} \leq p_{j}$ and $p_{j} \leq p_{k}$, then $p_{i} \leq p_{k}$. Thus $\leq$ is a partial order on $P$. Lemma 3.1 then guarantees that $G=G(P)$. Hence $G$ is Cohen-Macaulay, as desired.

Corollary 3.5 Let $G$ be a finite bipartite graph and $\Delta$ the simplicial complex whose StanleyReisner ring coincides with $I(G)$. Then $G$ is Cohen-Macaulay if and only if $\Delta$ is pure and strongly connected.

Work with the same situation as in the "if" part of the proof of Theorem 3.4. Let $\operatorname{com}(P)$ denote the comparability graph of $P$, i.e., $\operatorname{com}(P)$ is the finite graph on $\left\{p_{1}, \ldots, p_{n}\right\}$ whose edges are those $\left\{p_{i}, p_{j}\right\}$ with $i \neq j$ such that $p_{i}$ and $p_{j}$ are comparable in $P$. It then follows from [15, pp. 184-185] that the Cohen-Macaulay type of the Cohen-Macaulay ring $S / I(G)$, where $S=K\left[x_{1}, \ldots, x_{n}, y_{1}, \ldots, y_{n}\right]$, is the number of maximal independent subsets of $\operatorname{com}(P)$, i.e., the number of maximal antichains of $P$. Hence $G$ is Gorenstein, i.e., $S / I(G)$ is a Gorenstein ring, if and only if $P$ is an antichain.

Corollary 3.6 A Cohen-Macaulay bipartite graph $G$ is Gorenstein if and only if $G$ is the disjoint union of edges. 


\section{References}

1. D. Bayer and B. Sturmfels, "Cellular resolutions of monomial modules," J. Reine Angew. Math. 502 (1998), $123-140$.

2. S. Blum, "Subalgebras of bigraded Koszul algebras," J. Algebra 242 (2001), 795-809.

3. W. Bruns and J. Herzog, Cohen-Macaulay Rings, Revised Edition, Cambridge University Press, 1996.

4. J. Eagon and V. Reiner, "Resolutions of Stanley-Reisner rings and Alexander duality," J. Pure Appl. Algebra 130 (1998), 265-275.

5. D. Eisenbud, Commutative Algebra with a View Toward Algebraic Geometry, Springer-Verlag, New York, NY, 1995.

6. R. Fröberg, Koszul algebras, "Advances in commutative ring theory" in D.E. Dobbs, M. Fontana and S.-E. Kabbaj (Eds.), Lecture Notes in Pure and Appl. Math., Vol. 205, Dekker, New York, NY, 1999, pp. 337-350.

7. J. Herzog, T. Hibi, and X. Zheng, "Dirac's theorem on chordal graphs and Alexander duality," European J. Comb. 25(7) (2004), 826-838.

8. J. Herzog, T. Hibi, and X. Zheng, "The monomial ideal of a finite meet semi-lattice," to appear in Trans. AMS.

9. T. Hibi, "Distributive lattices, affine semigroup rings and algebras with straightening laws," in Commutative Algebra and Combinatorics, Advanced Studies in Pure Math., M. Nagata and H. Matsumura, (Eds.), Vol. 11, North-Holland, Amsterdam, 1987, pp. 93-109.

10. T. Hibi, Algebraic Combinatorics on Convex Polytopes, Carslaw, Glebe, N.S.W., Australia, 1992.

11. C. Peskine and L. Szpiro, "Syzygies and multiplicities," C.R. Acad. Sci. Paris. Sér. A 278 (1974), 1421-1424.

12. R.P. Stanley, Enumerative Combinatorics, Vol. I, Wadsworth \& Brooks/Cole, Monterey, CA, 1986.

13. R.P. Stanley, Combinatorics and Commutative Algebra, Second Edition, Birkhäuser, Boston, MA, 1996.

14. B. Sturmfels, "Gröbner Bases and Convex Polytopes," Amer. Math. Soc., Providence, RI, 1995.

15. R.H. Villareal, Monomial Algebras, Dekker, New York, NY, 2001. 